\title{
Guías chilenas de fibrosis pulmonar idiopática, 2019
}

\author{
Chilean clinical practice guidelines for idiopathic pulmonary fibrosis, 2019
}

La fibrosis pulmonar idiopática (FPI) es una enfermedad pulmonar intersticial (EPI) fibrosante progresiva y finalmente fatal de causa desconocidal. Es una de las EPI más comunes, grupo que también incluye a la neumonitis por hipersensibilidad (NHS) y a las EPI asociadas a las enfermedades del tejido conectivo (EPI-ETC), entre otras.

Aunque el curso es variable, su progresión se caracteriza por el deterioro de la función pulmonar, de la disnea, de la capacidad de ejercicio y de la calidad de vida. Las hospitalizaciones son comunes en pacientes con FPI, con tasas de mortalidad altas.

Publicaciones sobre pacientes chilenos con FPI nos muestran que la enfermedad se ve principalmente en hombres, la mayoría sobre 60 años, recurriendo a confirmación con biopsia pulmonar quirúrgica en $18 \%$ de los casos ${ }^{2}$. La mediana de supervivencia de una cohorte chilena del Instituto Nacional del Tórax de 142 pacientes con FPI confirmados con biopsia fue de 80 meses (RIQ 35-117)3.

La tasa de mortalidad por FPI en Chile ha ido aumentando progresivamente desde 18,5 x 100.000 en 2002 a 24,6 100.000 el año 2015, con diferencias regionales significativas como la tasa muy elevada que se observa en la XV región (Arica-Parinacota).

\section{¿Está aumentando la incidencia de la FPI en el mundo?}

A simple vista pareciera que sí. Basta considerar en nuestros hospitales el número de pacientes ingresados por exacerbaciones o por necesidad de procedimientos en estos días, con los que veíamos hace algunos años atrás, o bien el número de pacientes ambulatorios que controlamos, para afirmarlo. Sin embargo, esto pudiera tener otras explicaciones, siendo tal vez la más importante, la mejoría en los medios diagnósticos y el mejor conocimiento de la enfermedad de los neumólogos en los últimos años. Hay, sin embargo, estudios en el Reino Unido y en los Estados Unidos ${ }^{6}$ que lo demuestran.

Lo que si es claro es que la incidencia de la enfermedad aumenta dramáticamente con la edad y en cada decenio la prevalencia y la incidencia de la enfermedad crece.

\section{¿Por qué se produce la enfermedad?}

En los últimos años ha habido un aumento vertiginoso de las publicaciones sobre FPI en todos sus aspectos. Se conoce que hay claras alteraciones genéticas que condicionan su aparición, y/o sus exacerbaciones ${ }^{7}$ cuando ciertos promotores actúan ${ }^{8}$. Esto ocurre especialmente en el envejecimiento normal donde se producen cambios moleculares que condicionan su aparición ${ }^{9}$. Básicamente se trata de una alteración del mecanismo de reparación de la membrana basal alveolar sometida a múltiples y repetidas microlesiones. Se produciría un fenómeno por el cual varias células pulmonares desarrollan conductas aberrantes llevando al desarrollo y la manutención del proceso fibrótico ${ }^{10}$.

Durante más de 20 años se utilizó en el mundo un tratamiento equivocado que sólo recientemente se demostró que, más que inútil, fue perjudicial, lo cual es dramático considerando el ominoso pronóstico de la enfermedad ${ }^{11}$. Lo aleccionador fue que todo ese error se derivó de una concepción equivocada del mecanismo de producción pues consideraba la fibrosis pulmonar como secundaria a un proceso inflamatorio y eso demoró décadas en investigación y trabajo para demostrar al fin que no era asi' ${ }^{12}$.

Recién en 2005 y 2009 los autores japoneses primero ${ }^{13,14}$ y posteriormente grandes ensayos multinacionales, dieron la gran noticia: contábamos con medicamentos que, cumpliendo todas las condiciones que exige la evidencia en medicina, eran eficaces en cambiar el curso de la enfermedad enlenteciendo el avance de la misma y permitiendo ganar años de vida a una mayoría importante de pacientes. Lo 
más notable es que casi simultáneamente aparecieron la Pirfenidona ${ }^{15,16}$ y el Nintedanib ${ }^{17}$, dos antifibróticos diferentes, ambos eficaces, y se ha abierto una gama de nuevas moléculas que se están probando y seguramente en los próximos años dispondremos de nuevas drogas útiles ${ }^{18}$.

Lamentablemente los pacientes con FPI sufren un desamparo especial en Chile debido a que la enfermedad no está cubierta por ninguna ley de salud extraordinaria (AUGE o Ricarte Soto) y el costo de estos medicamentos le impide a la gran mayoría de los pacientes adquirirlos. Pensamos que esto debe cambiar ya que es dramático no poder ofrecer a nuestros pacientes un medicamento útil y no contar con otra alternativa de terapia eficaz. Agrava esta realidad el hecho demostrado que muchos pacientes son enviados tardíamente al especialista y que aún en manos de éstos, el diagnóstico demora varios meses en establecerse y está claro que tiene fundamental importancia el inicio precoz de tratamiento para la mayor supervivencia de los pacientes ${ }^{19,20}$.

La presente guía chilena de manejo de esta enfermedad, en la cual han colaborado un importante número de neumólogos chilenos, es un primer paso y el segundo es conseguir la posibilidad que estos nuevos medicamentos puedan ser accesibles precozmente para los pacientes portadores de esta terrible $y$ fatal enfermedad.

Dr. Álvaro Undurraga P. Profesor Adjunto Universidad de Chile Instituto Nacional del Tórax. Clínica Las Condes Email: alvaroundurragap@yahoo.com

\section{Bibliografía}

1.- RAGHU G, REMY-JARDIN M, MYERS JL, RICHELDI L, RYERSON CJ, LEDERER DJ, et al. Diagnosis of idiopathic pulmonary fibrosis: an official ATS/ERS/JRS/ALAT clinical practice guideline. Am J Respir Crit Care Med 2018; 198: e44-e68.

2.- UNDURRAGA A, MONTECINOS L, FLORENZANO M, RODRÍGUEZ JC, SALINAS M, REYES $\mathrm{M}$, et al. Primer registro nacional de fibrosis pulmonar idiopática (FPI) en Chile. Caracterización epidemiológica de 700 pacientes reclutados. Rev Chil Enf Respir 2017; 33: S 47-S 70.

3.- SALINAS M, FLORENZANO M, SABBAGH E, MENESES M, FERNÁNDEZ C, JALILIE A, et al. Supervivencia de pacientes con fibrosis pulmonar idiopática diagnosticados por biopsia quirúrgica de pulmón: experiencia del Instituto Nacional del Tórax. Rev Med Chile 2014; 142: 9-15.

4.- MENDOZA L, CAVADA G, MATUS P, VALDÉS N. Idiopathic Pulmonary Fibrosis: Mortality Trends in Chile. En: Poster presentado en European Respiratory Society International Congress; 2019.

5.- NAVARATNAM V, FLEMING KM, WEST J, SMITH CJ, JENKINS RG, FOGARTY A, et al. The rising incidence of Idiopathic Pulmonary Fibrosis in UK. Thorax 2011; 66: 462-7.

6.- RAGHU G, WEYCKER D, EDELSBERG J, BRADFORF WZ, OSTER G. Incidence and prevalence of idiopathic pulmonary fibrosis. Am J Respir Crit Care Med 2006; 174: 810-6.
7.- SELMAN M, PARDO A, BARRERA L, ESTRADA A, WATSON SR, WILSON K, et al. Gene expression profiles distinguish idiopathic pulmonary fibrosis from hypersensitivity pneumonitis. Am J Respir Crit Care Med 2006; 173: 188-98.

8.- MARTÍNEZ FJ. Idiopathic Interstitial Pneumonias. Usual interstitial pneumonia versus nonspecific intertitial pneumonia. Proc Am Thorac Soc 2006; 3: 81-95.

9.- SELMAN M, LÓPEZ OTIN C, PARDO A. Agedriven developmental drift in the pathogenesis of idiopathic pulmonary fibrosis Eur Respir J 2016; 48: 538-52.

10.- SGALLA G, IOVENE B, CALVELLO M, ORI M, VARONE F, RICHELDI L. Idiopathic pulmonary fibrosis: pathogenesis and management. Respir Res 2018; 19: 32 .

11.- RAGHU G, ANSTROM KJ, KING TE JR, LASKY JA, MARTÍNEZ FJ; Idiopathic Pulmonary Fibrosis Clinical Research Network. Prednisone, azathioprine, and $\mathrm{N}$-acetylcysteine for pulmonary fibrosis. $\mathrm{N}$ Engl J Med 2012; 366: 1968-77.

12.- SELMAN M, KING TE, PARDO A. Idiopathic pulmonary fibrosis: prevailing and evolving hypothesis about its pathogenesis and implications for therapy. Ann Intern Med 2001; 134: 136-51.

13.- AZUMA A, NUKIWA T, TSUBOI E, SUGA M, ABE S, NAKATA K, et al. Double-blind placebo controlled trial of pirfenidone in patients with idiopathic pulmonary fibrosis. Am J Respir Crit Care Med 2005; 171: 1040-7.

14.- TANIGUCHI H, EBINA M, KONDOCH Y, OGURA 
T, AZUMA A, SUGA M, et al. Pirfenidone in idiopathic pulmonary fibrosis. Eur Respir J 2010; 35: 821-9.

15.- NOBLE PW, ALBERA C, BRADFORD WZ, COSTABEL U, GLASSBERG MK, KARDATSKE D, et al. Pirfenidone in patients with idiopathic pulmonary fibrosis (CAPACITY): two randomised trials. Lancet 2011; 377: 1790-9.

16.- KING TE, Jr, BRADFORD WZ, CASTROBERNARDINI S, FAGAN EA, GLASPOLE I, GLASSBERG MK, et al. A phase 3 trial of pirfenidone in patients with idiopathic pulmonary fibrosis. $\mathrm{N}$ Engl $\mathrm{J}$ Med 2014; 370: 2083-92.

17.- RICHELDI L, DU BOIS RM, RAGHU M, AZUMA A, BROWN KK, COSTABEL U, et al. Efficacy and safety of nintedanib in idiopathic pulmonary fibrosis. $\mathrm{N}$ Engl J Med 2014; 370: 2071-82.

18.- SOMOGYI S, CHAUDHURI N, TORRISI SE, KAHN N, MÜLLER V, KREUTER M, et al. The therapy of idiopathic pulmonary fibrosis: what is next? Eur Respir Rev 2019; 28:190021 DOI: 10.1183/16000617.0021-2019.

19.- LAMAS DJ, KAWUT SM, BAGIELLA E, PHILLIP N, ARCASOY SM, LEDERER DJ. Delayed access and survival in idiopathic pulmonary fibrosis: a cohort study. Am J Respir Crit Care Med 2011; 184: 842-7.

20.- MAHER TM, STREK ME. Antifibrotic therapy for idiopathic pulmonary fibrosis: time to treat. Respir Res 2019; 20: 205. 